\title{
ON MONOMIAL ALGEBRAS OF FINITE GLOBAL DIMENSION
}

\author{
BY \\ DAVID J. ANICK ${ }^{1}$
}

\begin{abstract}
Let $G$ be an associative monomial k-algebra. If $G$ is assumed to be finitely presented, then either $G$ contains a free subalgebra on two monomials or else $G$ has polynomial growth. If instead $G$ is assumed to have finite global dimension, then either $G$ contains a free subalgebra or else $G$ has a finite presentation and polynomial growth. Also, a graded Hopf algebra with generators in degree one and relations in degree two contains a free Hopf subalgebra if the number of relations is small enough.
\end{abstract}

1. Introduction. Connected graded algebras over a field (see [1] for definitions) arise naturally in several contexts, including as the cohomology ring of a space with coefficients in a field, the homology ring of an associative $H$-space, and the Yoneda Ext-algebra of a local Noetherian ring. The last two of these are in general noncommutative graded algebras, and a natural question arises in their study as to whether or not they contain free noncommutative graded subalgebras. In [4], Luchezar Avramov poses this question for certain Ext-algebras as a conjecture. Felix and Halperin [9] and others have used rational homotopy theory to explore this question for $H_{*}(\Omega X ; \mathbf{Q})$, where $X$ is a space of finite rational category.

Not all of what we prove in this article applies directly to local rings or to loop spaces, but there is a close connection. In many instances, such as when the cube of a ring's maximal ideal vanishes or when a space has rational category two, the question concerning free subalgebras reduces to the same question asked about a certain finitely presented (noncommutative) graded Hopf algebra $[15,10]$. In these and other settings we may also know that a graded algebra has finite global dimension, whether or not it is finitely presented. The most general question which can be asked along these lines is the following.

Question 1. Let $G$ be a connected, nonnegatively graded algebra (resp. Hopf algebra) over a field. Suppose either that $G$ is finitely presented or that gl.dim. $(G)<$ $\infty$. Is it true that $G$ must have either subexponential or polynomial growth, or else contain a free subalgebra (resp. Hopf subalgebra) on two homogeneous generators?

The restrictions on $G$ embedded in Question 1 are easy to explain. A free subalgebra on two homogeneous generators would have exponential growth, so if $G$ 's growth is subexponential it has no hope of containing a free subalgebra. Golod and Shafarevich [11] constructed finitely generated graded algebras with exponential

Received by the editors November 15, 1984.

1980 Mathematics Subject Classification. Primary 16A03, 16A60.

${ }^{1}$ Partially supported by NSF grant \# DMS-8303257. 
growth in which each element is nilpotent. These algebras do not even contain a free subalgebra on one generator, so clearly the condition of being finitely generated is not enough. Finally, a free algebra (resp. free Hopf algebra) on two elements automatically contains a free subalgebra on a countably infinite set of generators (resp. primitive generators), so there is no reason to look for subalgebras (resp. Hopf subalgebras) with three or more generators.

$\S \S 2$ and 3 of this paper deal with monomial $\mathbf{k}$-algebras. A monomial $\mathbf{k}$-algebra is an associative graded $\mathbf{k}$-algebra ( $\mathbf{k}$ is any field) which has a presentation as $G \approx \mathbf{k}\langle S\rangle /\langle W\rangle$, where $S$ denotes a graded set of generators and the ideal of relations is generated as a two-sided ideal by a collection $W$ of monomials in $S$. Monomial algebras are studied partly because they are the combinatorially simplest kind of graded algebras, so sharper theorems can be expected, and partly because problems for larger classes of algebras can sometimes be reduced to them. In particular, we plan to prove

THEOREM 1. Let $G$ be a monomial $\mathbf{k}$-algebra, and suppose either (a) that $G$ is finitely presented or (b) that gl.dim. $(G)<\infty$. Then either $G$ contains a free subalgebra on two monomials or $G$ has polynomial growth.

Part (a) will be proved as Theorem 3, and part (b) will be implicit in Theorem 6 .

The last section of the paper deals with graded Hopf algebras having generators in degree one and relations in degree two. These objects play a central role in the theory of local rings $(R, \mathbf{m}, \mathbf{k})$ having $\mathbf{m}^{3}=0[13]$, and also in the theory of $\Omega X$ for $X$ a 1 -connected CW complex with $\operatorname{dim}(X)=4[12,15]$. In particular, $\operatorname{Ext}_{R}^{*}(\mathbf{k}, \mathbf{k})$ contains a free subalgebra if a certain graded Hopf algebra $G$ does, where $G$ has $n=\operatorname{dim}\left(\mathbf{m} / \mathbf{m}^{2}\right)$ generators in degree one and $d=\operatorname{dim}\left(\mathbf{m}^{2}\right)$ relations in degree two. Theorem 9 includes the following.

THEOREM 2. Let $(R, \mathbf{m}, \mathbf{k})$ be a local ring with $\mathbf{m}^{3}=0$, where $\mathbf{k}$ is algebraically closed, $\operatorname{dim}\left(\mathbf{m} / \mathbf{m}^{2}\right)=n$ and $\operatorname{dim}\left(\mathbf{m}^{2}\right)=d$. If $d \leqslant \frac{1}{2}(3 n-5)$, then $\operatorname{Ext}_{R}^{*}(\mathbf{k}, \mathbf{k})$ contains a free Hopf subalgebra on two generators of degree one or two.

2. Finitely presented monomial algebras. In this section we prove that a finitely presented monomial algebra with exponential growth always contains free subalgebras.

We begin by describing a vector space basis for a monomial $\mathbf{k}$-algebra. A $\mathbf{k}$-basis for the free algebra $\mathbf{k}\langle S\rangle$ is $T=T_{S}$, the set of all monomials (also called words) on $S$, including the empty word denoted " 1 ". A monomial $u$ is nontrivial if $u \neq 1$. For $u$ and $v$ monomials, we say that $u$ is a left factor or initial segment (resp. proper left factor) of $v$ if and only if $v=u q$ for some monomial (resp. nontrivial monomial) $q$, and likewise for right factors or final segments. The word $u$ is a segment of $v$ if $u$ is a right factor of a left factor of $v$. The relation "is a segment of" is a partial order on $T$. 
A k-basis for the monomial algebra $G \approx \mathbf{k}\langle S\rangle /\langle W\rangle$ is

$$
M=\{u \in T \mid \text { no } w \in W \text { is a segment of } u\} .
$$

We shall always assume, without loss of generality, that the presentation $G \approx$ $\mathbf{k}\langle S\rangle /\langle W\rangle$ is minimal. This means that $W \cap S$ is empty and that $W$ is an antichain with respect to the "segment of" partial order.

Monomial rings admit as many independent gradings as they have generators, since the relations $W$ are automatically homogeneous. Changing from one (positive) grading to another does not affect the nature of the growth of $G$, as the following lemma shows. The situation for the growth of groups under a change of generators is analogous [14, Lemma 1].

LEMMA 1. Let $G \approx \mathbf{k}\left\langle x_{1}, \ldots, x_{g}\right\rangle /\left\langle w_{1}, w_{2}, \ldots\right\rangle$ be a finitely generated monomial $\mathbf{k}$-algebra with the set function $e:\left\{x_{1}, \ldots, x_{g}\right\} \rightarrow \mathbf{Z}_{+}$determining its grading. Let $G^{\prime}$ be an algebra with the same presentation as $G$, but let $G^{\prime}$ be graded by length, i.e., set each $\left|x_{i}\right|=1$. Then $G$ has polynomial growth if and only if $G^{\prime}$ has polynomial growth.

Proof. Let

$$
M=\{u \in T \mid \text { no segment of } u \text { is in } W\}
$$

be the collection of monomials which serves as a k-basis for both $G$ and $G^{\prime}$. Let $a_{n}=\#\{u \in M \mid e(u)=n\}$ and $b_{n}=\#\{u \in M|| u \mid=n$,$\} so the respective Hilbert$ series are

$$
H_{G}(z)=\sum_{n=0}^{\infty} a_{n} z^{n} \text { and } H_{G^{\prime}}(z)=\sum_{n=0}^{\infty} b_{n} z^{n} .
$$

Setting $m=\max \left\{e\left(x_{1}\right), \ldots, e\left(x_{g}\right)\right\}$, we have

$$
b_{n} \leqslant a_{n}+a_{n+1}+\cdots+a_{m n} \text { and } a_{m n-p} \leqslant b_{n}+b_{n+1}+\cdots+b_{m n-p}
$$

whenever $0 \leqslant p<m$. If $a_{n} \leqslant c n^{d}$ for all $n$, then

$$
b_{n} \leqslant \sum_{i=n}^{m n} c i^{d} \leqslant\left(c m^{d}\right) n^{d}(m n-n+1) \leqslant c^{\prime} n^{d+1}
$$

for some constant $c^{\prime}$. Likewise $b_{n} \leqslant c n^{d}$ for all $n$ leads to $a_{n} \leqslant c^{\prime} n^{d+1}$ for some $c^{\prime}$. This is the desired conclusion.

A more subtle argument shows that the least exponents $d$ and $d^{\prime}$ governing the polynomial rates of growth for $G$ and $G^{\prime}$ must actually coincide. For our purposes, however, Lemma 1 suffices: we may assume that all $e\left(x_{i}\right)=1$ in proving the following theorem.

THEOREM 3. Let

$$
G \approx \mathbf{k}\left\langle x_{1}, \ldots, x_{g}\right\rangle /\left\langle w_{1}, \ldots, w_{r}\right\rangle
$$

be any finitely presented connected graded monomial $\mathbf{k}$-algebra. Then either $G$ contains a free subalgebra on two monomials, or else $G$ has polynomial growth.

Proof. As mentioned, we may by Lemma 1 assume that $G$ is graded so that each $\left|x_{i}\right|=1$. Write $W=\left(w_{1}, \ldots, w_{r}\right)$ and let $d=\max \left\{\left|w_{i}\right| \mid w_{i} \in W\right\}$. Let $M$ be the k-basis for $G$ described above and let

$$
L=\left\{u \in T|| u \mid=d \text { and no } w_{i} \text { is a segment of } u\right\},
$$


which is a $\mathbf{k}$-basis for the degree $d$ part of $G$. For $u, v \in L$, write " $u \vdash v$ " if $u v \in M$ and write " $u \vDash v$ " if there exists a sequence $u_{1}, \ldots, u_{p}$ in $L$ with $p \geqslant 2$ and $u=u_{1} \vdash u_{2} \vdash \cdots \vdash u_{p}=v$. Note that this condition implies that the product $u_{1} u_{2} \cdots u_{p}$ lies in $M$ because each $w_{i} \in W$ could cross at most one "boundary" between successive factors in that product.

One of these two mutually exclusive cases must be true for $G$ :

(A) There exist $u, v, y \in L, v \neq y$, for which $u \vdash v \vDash u$ and $u \vdash y \vDash u$.

(B) For each $u \in L$, there is at most one $v \in L$ such that $u \vdash v$ and $v \vDash u$.

In case (A), we write $u \vdash v=v_{1} \vdash v_{2} \vdash \cdots \vdash v_{p}=u$ and $u \vdash y=y_{1} \vdash y_{2} \vdash$ $\cdots \vdash y_{q}=u$. Then the subalgebra on $b_{1}=v_{1} v_{2} \cdots v_{p}$ and $b_{2}=y_{1} y_{2} \cdots y_{q}$ is free in $G$.

In case (B), define an equivalence relation on $L$ by $u \sim v$ if and only if $u=v$ or $u \vDash v \vDash u$. Clearly $u_{1} \sim u_{2} \vDash v_{2} \sim v_{1}$ implies $u_{1} \vDash v_{1}$, so the relation $\vDash$ becomes a partial order on equivalence classes. Write " $u \downarrow v$ " if $u \vDash v$ but $u+v$. Let

$$
a_{n, u}=\#\{y \in M|| y \mid=n d \text { and } u \text { is a left factor of } y\} .
$$

Then

$$
a_{n, u}=\#\left\{\text { sequences } u=u_{1} \vdash u_{2} \vdash \cdots \vdash u_{n} \mid u_{j} \in L\right\},
$$

so $a_{n, u}=\sum_{u \vdash v} a_{n-1, v}$ for $n \geqslant 2$, while $a_{1, u}=1$ for all $u \in L$. In view of condition (B), there exists for each $u \in L$ at most one monomial $u^{\prime} \in L$ for which $u \vdash u^{\prime} \vDash u$. If such a $u^{\prime}$ does exist for a particular $u$, then

$$
a_{n, u} \leqslant a_{n-1, u^{\prime}}+\sum_{u \downarrow u} a_{n-1, \nu^{\prime}}
$$

If for a particular $u$ there is no $u^{\prime}$ satisfying $u \vdash u^{\prime} \vDash u$, then formula (1) is still true if we interpret the term $a_{n-1, u^{\prime}}$ as zero.

Staying wtih case (B) above, we claim that the formula

$$
a_{n, u} \leqslant 1+\sum_{i=1}^{n-1} \sum_{u \downarrow v} a_{i, v}
$$

is valid for all $n$ and $u$. We have already observed that (2) holds for $n=1$. Assuming (2) holds for some particular $n$, we have by (1)

$$
\begin{aligned}
a_{n+1, u} & \leqslant a_{n, u^{\prime}}+\sum_{u \downarrow v} a_{n, v} \\
& \leqslant 1+\sum_{i=1}^{n-1}\left(\sum_{u^{\prime} \downarrow v} a_{i, v}\right)+\sum_{u \downarrow v} a_{n, v} \\
& \leqslant 1+\sum_{i=1}^{n} \sum_{u \downarrow v,} a_{i, v},
\end{aligned}
$$

as desired.

The fact that equivalence classes are partially ordered under $\vDash$ and that there are at most $\#(L)$ classes means that we can label the classes as $\alpha_{1}, \alpha_{2}, \ldots, \alpha_{c}$, where $\alpha_{i} \vDash \alpha_{j}$ only if $i \geqslant j$. Let $t_{0}=1$ and for $1 \leqslant j \leqslant c$ let $t_{j}$ denote the product 
$\#\left(\alpha_{1}\right) \#\left(\alpha_{2}\right) \cdots \#\left(\alpha_{j}\right)$. By (2) we have $a_{n, u} \leqslant 1$ for $u \in \alpha_{1}$, and we assert that

$$
a_{n, u} \leqslant t_{m-1}\left(\begin{array}{c}
n+m-2 \\
m-1
\end{array}\right) \quad \text { for } u \in \alpha_{m}
$$

To prove (3), note that we have already observed that it holds for $m=1$, so suppose (3) holds for some $m$. Then for $u \in \alpha_{m+1}$, we have by formula (2)

$$
\begin{aligned}
a_{n, u} & \leqslant 1+\sum_{i=1}^{n-1} \sum_{u \downarrow v} a_{i, v} \leqslant 1+\sum_{i=1}^{n-1} \sum_{j=1}^{m} \sum_{v \in \alpha_{j}}\left(\begin{array}{c}
i+j-2 \\
j-1
\end{array}\right) t_{j-1} \\
& =1+\sum_{i=1}^{n-1} \sum_{j=1}^{m}\left(\begin{array}{c}
i+j-2 \\
j-1
\end{array}\right)\left(t_{j-1} \#\left(\alpha_{j}\right)\right) \\
& =1+\sum_{i=1}^{n-1} \sum_{j=1}^{m}\left(\begin{array}{c}
i+j-2 \\
j-1
\end{array}\right) t_{j} \leqslant 1+\left(t_{m}\right) \sum_{i=1}^{n-1} \sum_{j=1}^{m}\left(\begin{array}{c}
i+j-2 \\
j-1
\end{array}\right) \\
& =1-t_{m}+t_{m}\left(\begin{array}{c}
m+n-1 \\
m
\end{array}\right) \leqslant\left(\begin{array}{c}
m+n-1 \\
m
\end{array}\right) t_{m},
\end{aligned}
$$

as desired. In particular, we may conclude that

$$
a_{n, u} \leqslant t_{c-1} n^{c-1}
$$

for any $u \in L$.

From (4) we get that

$$
\begin{aligned}
\#\{y \in M|| y \mid= & n d+s\} \leqslant\left(g^{s}\right) \#\{y \in M|| y \mid=n d\} \\
& =\left(g^{s}\right) \sum_{u \in L} a_{n, u} \leqslant\left(g^{d-1} t_{c-1}\right) \#(L) n^{c-1} \\
& =(\text { constant }) n^{c-1} \quad \text { for } 0 \leqslant s<d,
\end{aligned}
$$

so $G$ has polynomial growth.

3. Monomial algebras with finite global dimension. We turn our attention now to algebras with finite global dimension. The condition of finite global dimension is, in many respects, more stringent than the condition of finite presentation. It will turn out that a monomial algebra with finite global dimension but without free subalgebras is necessarily a finitely presented algebra of a very special kind.

To work with the concept of finite global dimension, we recall from [5] a computation of $G$ 's homology. The graded algebra $G$ is no longer assumed to be finitely generated. The computation is best described in terms of "chains" and "prechains", so we define these terms next.

DEFinition. With a set of generators $S$, the free monoid $T$ on $S$, and an antichain $W \subseteq T$ as in $\S 2$, define the set of $n$-chains recursively, as follows. A (-1)-chain is the monomial 1 , a 0 -chain is any element of $S$, and a 1-chain is a word in $W$. An $(n+1)$-prechain is a word $w \in T$ which has two factorizations $w=u v q=u s t$ in $T$, where $u$ is an $(n-1)$-chain, $s$ is a proper left segment of $v, u v$ is an $n$-chain, and $t \in W$. The right factor $q$ is called the tail of the prechain. An $(n+1)$-prechain $w$ is an $(n+1)$-chain if and only if no proper left segment of $w$ is also an $(n+1)$-prechain.

To illustrate the concepts of chains and prechains we offer two examples. 
ExAmple 1. Suppose $S=\{a, b, c\}$ and $W=\{a b c a, c a b\}$. The 2-prechains are $w_{21}=a b c a b c a, w_{22}=a b c a b$, and $w_{23}=c a b c a$. Each 2-prechain consists of two (possibly identical) words from $W$ which overlap. For example, the two factorizations associated with $w_{23}$ are

$$
w_{23}=(c)(a b)(c a)=(c)(1)(a b c a) \text {. }
$$

Of the three 2-prechains, only $w_{22}$ and $w_{23}$ are 2-chains, since $w_{21}$ has $w_{22}$ as a proper left segment. The 3-prechains are

$$
w_{31}=(c a b)(c a)(b c a)=(c a b)(c)(a b c a)
$$

and

$$
w_{32}=(c a b)(c a)(b)=(c a b)(1)(c a b),
$$

of which only $w_{32}$ is a 3-chain. There are no 4-prechains by the following reasoning. Any 4-prechain $w$ would require a 3-chain as a left factor, but we have only one 3-chain, so $w$ would have to look like $w=\left({ }^{\prime} c a b c a\right)(b)(x)$ for some word $x$. The "ust" factorization of $w$ requires that $b x$ equal st for some $t \in W$ while $s$ must be a proper subword of the monomial $b$, forcing $s=1$ and $b x \in W$. But no words in $W$ start with the letter $b$. Thus we have found all the $n$-chains for this set $W$ of monomials.

Example 2. To show how subtly the set of $n$-chains depends upon $W$, we alter the previous example by just one letter, keeping $S=\{a, b, c\}$ but setting $W=$ $\{a b c a, a a b\}$. The 2-prechains are aabca, abcabca, and abcaab; all of these are 2-chains. By trying various tails to append to each of these 2-chains we can identify all 3-prechains. Writing a 3-prechain in the form $(u \mathbf{v}) q$, our list is: (aabca)ab, ( $a a b \mathbf{c a}) b c a$, ( $a b c a \mathbf{b c a}) a b$, (abcabca) $b c a$, ( $a b c a \mathbf{a b}) c a$. All of these are in fact 3-chains. As an exercise, the reader is encouraged to verify that for this example the number of $n$-chains equals the $(n+2)$ nd Fibonacci number when $n \geqslant 1$.

The next theorem indicates why we are interested in the concept of $n$-chains.

THEOREM 4. Let $W$ be an antichain of monomials in the free monoid on the graded set $S$. If $G=\mathbf{k}\langle S\rangle /\langle W\rangle$ and $i \geqslant 0$, then $\operatorname{Tor}_{i, *}^{G}(\mathbf{k}, \mathbf{k})$ is isomorphic as a graded k-module with the span of the set of $(i-1)$-chains for $W$. In particular, gl.dim. $(G) \leqslant d$ if and only if there are no d-chains (equivalently, no d-prechains).

ProOF. See [5 or 2, Lemma 3.3].

Our goal is a theorem which will characterize quite crisply the nature of a monomial algebra with finite global dimension and without free subalgebras. The bulk of the proof will take place in the context of a fairly lengthy induction argument. The next four paragraphs offer notations and definitions which will be essential in that induction.

For $u, v \in T$, let $F_{u, v}$ denote the submonoid of $T$ generated by $u$ and $v$, and let $\langle u, v\rangle$ denote the collection of all segments of words in $F_{u, v}$. The condition that $G$ contain no free subalgebra on two monomials is equivalent to the requirement that $W \cap\langle u, v\rangle$ be nonempty whenever $F_{u, v}$ is free on two generators. A sufficient condition that $F_{u, v}$ be free on two generators is that $u \neq v$ and $u, v \in V$, where

$$
V=\left\{u \in T \mid u \neq w^{l} \text { for any } l \geqslant 2 \text { and } w \in T\right\} \text {. }
$$


The sufficiency of this condition will be proved in Lemma 2 of $\S 4$; for now we assume it.

For $n \geqslant 0$ an integer and $r \in T$, write $n \varangle r$ if and only if, for some $l \geqslant n$, there is an $l$-chain $w$ such that $r$ is a right factor of its tail. Write $n \nabla r$ if and only if $n \varangle q$ for some nontrivial left factor $q$ of $r$. Thus $n \triangleleft q$ implies $n \triangleright q$ but not conversely, while $n \varangle q$ implies $n \varangle r$ for any right factor $r$ of $q$.

Write $u \rightarrow v$ if and only if there is a word

$$
w=s_{1} s_{2} s_{3} \in W \cap\langle u, v\rangle,
$$

where $s_{1}$ is a nontrivial right factor of $u, s_{2} \in F_{u, v}$, and $s_{3}$ is a left factor of $u$ or of $v$. The symbols $u=\rightarrow v$ mean $u \rightarrow v$ or $u=v$. The assumption of no free subalgebra implies, whenever $u, v \in M \cap V$ with $u \neq v$, that either $u \rightarrow v$ or $v \rightarrow u$, or both. (Recall from $\S 2$ that $M$ denotes the set of monomials containing no member of $W$ as a segment and that $M$ serves as a k-basis for the algebra $G$.)

Let $|u|$ denote the length of a monomial $u \in T$. If $G$ starts out with a different grading, it will not affect the upcoming Theorem 5 . Recursively define the collection of atoms for $G$ to be $A=\bigcup_{n=1}^{\infty} A_{n}$, where $A_{1}=S$ and, given $A_{n-1}, A_{n}$ is defined by

$$
A_{n}=A_{n-1} \cup\left\{u v \mid u \in A_{n-1}, v \in A_{n-1}, u \rightarrow v, u v \in M \text {, and }|u v|=n\right\} \text {. }
$$

The best insights into the algebraic structure of $G$ are afforded by understanding the behavior of its atoms. Theorem 5 does this in a big way, offering ten separate facts about $M$ and $A$. The most important fact for later use is unique factorization, while for the proof itself, chain extension and total ordering are the most vital.

THEOREM 5. With notations as above, suppose that the monomial algebra $G$ has finite global dimension but does not contain a free subalgebra on two monomials. Then the following ten propositions are true for all $n \geqslant 1$.

$\mathrm{U}_{n}$ (Unique factorization): Any word $w \in M-\{1\}$ with $|w| \leqslant n$ may be uniquely factored into $w=a_{1} a_{2} \cdots a_{m}$, where $a_{i} \in A_{n}$ and $a_{m}=\rightarrow \cdots=\rightarrow a_{2}=\rightarrow a_{1}$.

$\mathrm{S}_{n}$ (Segmentation): Let $u \in A_{n}$, and suppose $u=q r$, where $q \neq 1$ and $r \neq 1$. Suppose $q=a_{1} \cdots a_{m}$, where $a_{i} \in A_{n}$ and $a_{m}=\rightarrow \cdots=\rightarrow a_{1}$, and likewise for $r=b_{1} \cdots b_{l}$. Then there exist $s, t \in A_{n-1}$ with $u=s t$, for which $a_{m}=\rightarrow s \rightarrow t=\rightarrow$ $b_{1}$.

$\mathrm{P}_{n}$ (No proper powers): $A_{n} \subseteq V$. No atom is a proper power of a monomial.

$I_{n}$ (Interpolation): If $u, v \in A_{n-1}$ and $u \neq v$ and $u \rightarrow v$, and $u v \in M$ with $|u v| \leqslant n$, then either $u \rightarrow u v \rightarrow v$ or there exists $u^{\prime} \in A_{n-1}$ with $u \rightarrow u^{\prime} \rightarrow u v \rightarrow v$.

$\mathrm{F}_{n}$ (Full endings): If $m \triangleright u$ and $u \in A_{n}$, then $m \varangle u$.

$\mathrm{C}_{n}$ (Chain extension): If $u \in A_{n}, m \varangle u$, and $q \neq u$ is any monomial in $M$ with $u \rightarrow q$, then $(m+1) \triangleright q$.

$\mathrm{E}_{n}$ (Exclusivity): For $u, v \in A_{n}$, exactly one of the relations $u \rightarrow v, u=v$, and $v \rightarrow u$ is valid.

$\mathrm{T}_{n}$ (Total ordering): The relation $\rightarrow$ is a total order on $A_{n}$.

$\mathrm{L}_{n}$ (Left and right segments): If $u \in A_{n}$ and $v \in A_{n}$ and $v$ is a left (resp. right) factor of $u$, then $v=\rightarrow u($ resp. $u=\rightarrow v)$.

$\mathbf{R}_{n}$ (The relations in $W$ ): Any word $w \in W$ with $|w| \leqslant n$ is a "would-be-atom." That is, it equals $u v$ for some $u, v \in A_{n-1}$ having $u \rightarrow v$. 
Proof. We fix $n$ and suppose that either $n=1$ or that all ten propositions are known to hold for $n-1$. In particular, we shall make free use of $T_{n-1}$ without noting each such application explicitly.

To prove proposition $\mathrm{U}_{n}\left(\mathrm{U}_{1}\right.$ is trivial) let $w \in M$ have $|w|=n$ and write $w=x v$, where $|x|=1$ and $v \in M$. Uniquely factor $v$ via proposition $\mathrm{U}_{n-1}$ as $v=b_{1} \cdots b_{m}$, where $b_{i+1}=\rightarrow b_{i}$. Let $i$ be the largest index for which $y_{i}=x b_{1} \cdots b_{i}$ is an atom ( $i=0$ and $i=m$ are both possible). Then the relation $y_{i} \rightarrow b_{i+1}$ would contradict the maximality of $i$, so by proposition $\mathrm{E}_{n-1}$ we have $b_{i+1}=\rightarrow y_{i}$. Consequently $y_{i} b_{i+1} \cdots b_{m}$ is a valid factorization of $w$.

For uniqueness, we first make a certain observation. Whenever $q$ is a word of length $n-1$ or less with unique factorization $q=a_{1} \cdots a_{l}$ and whenever $p$ is a left factor of $q$, then $p=a_{1} \cdots a_{i-1} r$ for some $i$ and for some nontrivial left segment $r$ of $a_{i}$. The observation is that, if $r$ is uniquely factored as $r=b_{1} \cdots b_{m}$, then the unique factorization of $p$ is $p=a_{1} \cdots a_{i-1} b_{1} \cdots b_{m}$. To see this it suffices to check that $b_{1}=\rightarrow a_{i-1}$. But this follows from proposition $\mathrm{L}_{n-1}$, which gives $b_{1}=\rightarrow a_{i}$ $=\rightarrow a_{i-1}$, to which we apply $\mathrm{T}_{n-1}$. Similarly, if $|q| \leqslant n-1$ and $p$ is a right factor of $q$ and $q=a_{1} \cdots a_{l}$ with $p=r a_{i+1} \cdots a_{l}$, then the unique factorization of $p$ is $p=b_{1} \cdots b_{m} a_{i+1} \cdots a_{l}$.

Now let $w \in A_{n}$, and suppose that $w=a_{1} \cdots a_{l}$ and $w=b_{1} \cdots b_{m}$ are two distinct monotonic atomic factorizations of $w$. Without loss of generality we may take $\left|a_{1}\right| \geqslant\left|b_{1}\right|$, and $\left|a_{1}\right|=\left|b_{1}\right|$ would imply the existence of two factorizations for $a_{2} \cdots a_{l}$, contradicting $\mathrm{U}_{n-1}$, so $\left|a_{1}\right|>\left|b_{1}\right|$. By the above observation, however, this would lead to two factorizations for $a_{1}$, contradicting $\mathrm{U}_{n-1}$ unless $l=1$. When $l=1$, factor $w=a_{1}$ as $w=u v$, where $u \in A_{n-1}, v \in A_{n-1}$, and $u \rightarrow v$. The above observation together with $\mathrm{U}_{n-1}$ guarantees that $|u| \leqslant\left|b_{1}\right|$ and that $|v| \leqslant\left|b_{m}\right|$. If $m \geqslant 2$, then

$$
\left|b_{1}\right|+\left|b_{m}\right| \geqslant|u|+|v|=|w|=\left|b_{1} \cdots b_{m}\right| \geqslant\left|b_{1}\right|+\left|b_{m}\right|
$$

so $m=2$ and equality holds throughout. But then $v=b_{2}=\rightarrow b_{1}=u \rightarrow v$, a contradiction.

To verify proposition $\mathrm{S}_{n}$ ( $\mathrm{S}_{1}$ is vacuous), let $u \in A_{n}$ and let $u=q r$ be any nontrivial factorization. Write $q=a_{1} \cdots a_{m}$ and $r=b_{1} \cdots b_{l}$, in accordance with $\mathrm{U}_{n-1}$. If $m=l=1$, then $q \rightarrow r$ and we are done (the possibility $r=\rightarrow q$ would yield two atomic factorizations of $u=q r$, contradicting the now established proposition $\mathrm{U}_{n}$ ). Otherwise construct a sequence $v_{1}, v_{2}, \ldots, v_{l+m}$ of atoms as follows. Start with $v_{1}=a_{m}$, and if for some $j$ there are indices $i$ and $p$ such that $u=a_{1} \cdots a_{i} v_{j} b_{p} \cdots b_{l}$, then either $a_{i} \rightarrow v_{j}$ or $v_{j} \rightarrow b_{p}$ (or both), since otherwise we would have two atomic factorizations of $u$. Define $v_{j+1}$ to be $a_{i} v_{j}$ if $a_{i} \rightarrow v_{j}$ and let $v_{j+1}=v_{j} b_{p}$ otherwise. The relation $a_{m} \rightarrow v_{2}=a_{m} b_{1} \rightarrow b_{1}$ is a consequence of $\mathrm{I}_{n-1}$, and by induction on $j$ we get that $a_{m} \rightarrow v_{j} \rightarrow b_{1}$. The last entry in our sequence must be $v_{l+m}=u$ and the penultimate entry $v_{l+m-1}$ must equal either $a_{1} \cdots a_{m} b_{1} \cdots b_{l-1}$ or $a_{2} \cdots a_{m} b_{1}$ $\cdots b_{l}$. In the former case use $s=v_{l+m-1}$ and $t=b_{l}$, yielding $a_{m} \rightarrow v_{l+m-1}=s \rightarrow t$ $=b_{l}=\rightarrow b_{1}$, and in the latter case choose $s=a_{1}$ and $t=v_{l+m-1}$, giving $a_{m}=\rightarrow a_{1}$ $=s \rightarrow t=v_{l+m-1} \rightarrow b_{1}$. 
For proposition $\mathrm{P}_{n}$ ( $\mathrm{P}_{1}$ is vacuous), if $|u|=n$ and $u=w^{\prime}$ with $l \geqslant 2$ we must prove that $u$ is not also an atom. Using $\mathrm{U}_{n}$ write $w=a_{1} \cdots a_{m}$, where $a_{m}=\rightarrow \cdots=\rightarrow a_{1}$, so $u=a_{1} \cdots a_{m} a_{1} \cdots a_{m} \cdots a_{m}$. Define a finite sequence of factorizations of $u$, $u=b_{i, 1} b_{i, 2} \cdots b_{i, l m+1-i}$ for $i=1,2, \ldots$, where $b_{i, j} \in A_{n}$ as follows. The first factorization in the sequence is the original one,

$$
u=b_{1,1} \cdots b_{1, l m}=a_{1} \cdots a_{m} a_{1} \cdots a_{m} \cdots a_{m} .
$$

Having found the $i$ th factorization, if it turns out that $b_{i, l m+1-i}=\rightarrow b_{i, l m-i}=\rightarrow$ $\cdots=\rightarrow b_{i, 1}$, then the $i$ th factorization is the last in the sequence. If instead any $b_{i, p} \rightarrow b_{i, p+1}$, let $p_{0}=p_{0}(i)$ be the first spot where this occurs and set

$$
b_{i+1, j}= \begin{cases}b_{i, j} & \text { for } 1 \leqslant j \leqslant p_{0} \\ b_{i, j} b_{i, j+1} & \text { for } j=p_{0} \\ b_{i, j+1} & \text { for } p_{0} \leqslant j \leqslant l m-i .\end{cases}
$$

Inductively we see by $\mathrm{I}_{n-1}$ that $b_{i, j}=\rightarrow a_{1}$ for all $i$ and $j$, so in particular $p_{0}(i)$ never equals 1 and $b_{i, 1}=a_{1}$ for all $i$. The last factorization in the sequence must occur while $i<\operatorname{lm}$, which means that $u$ has a monotonic atomic factorization as a product of two or more atoms. By $\mathrm{U}_{n}, u$ is not also an atom.

The proof of interpolation is quite technical and is postponed to the appendix.

For proposition $\mathrm{F}_{n}$ (trivial for $n=1$ ), suppose $m \nabla u$ and $|u|=n$. Let $r \neq 1$ be a left factor of $u$ for which $m \varangle r$. If $|r|=n$ we are done, so supposing $|r|<n$, factor $r$ using $\mathrm{U}_{n-1}$ into $r=a_{1} \cdots a_{l}$. Then $m \varangle a_{l}$ also holds, while by segmentation and interpolation $a_{l}=\rightarrow s=\rightarrow s^{\prime} \rightarrow u$ for some $s \in A_{n-1}$ and $s^{\prime} \in A_{n-1}$. Through $\mathrm{C}_{n-1}$ we get $m \triangleright s^{\prime}$, by $\mathrm{F}_{n-1}$ we get $m \varangle s^{\prime}$, and from $\mathrm{C}_{n-1}$ we obtain $(m+1) \triangleright u$. We are back where we started, but with a longer chain. Because $G$ has finite global dimension, this process cannot continue forever. Conclude that, at some point, $m \varangle u$ must occur.

The chain extension property (proposition $\mathrm{C}_{n}$ ) is one of the most important. Assuming $m \varangle u$ and $u \rightarrow q$ with $u \in A_{n}$, let $c u$ be any $m$-chain which has $u$ as a right factor of its tail and let $r w \in W \cap\langle u, q\rangle$ be a word having a nontrivial right factor $r$ of $u$ as an initial segment. The word $c u w$ is an $(m+1)$-prechain which must either be or contain as a left segment an $(m+1)$-chain of the form cus. This $(m+1)$-chain, with tail $s \in\langle u, q\rangle$, shows that either $(m+1) \nabla q$ or $(m+1) \nabla u$. If $(m+1) \nabla u$, use the now proved proposition $\mathrm{F}_{n}$ to conclude that $(m+1) \varangle u$, and we are back where we started, but with a longer chain. As before, the global dimension condition guarantees that eventually $(m+1) \triangleright q$ will occur.

Exclusivity and the total ordering flow from acyclicity, the condition that a cycle $u_{1} \rightarrow u_{2} \rightarrow \cdots \rightarrow u_{l} \rightarrow u_{1}$ cannot occur in $A_{n}$. To prove this, note that $u_{1} \rightarrow u_{2}$ says that either $(1) \triangleright u_{1}$ or $(1) \triangleright u_{2}$. If $(1) \triangleright u_{1}$, use $F_{n}$ and $C_{n}$ to obtain $(1) \triangleright u_{2}$, so (1) $\triangleright u_{2}$ in any case. By repeated application of $F_{n}$ and $C_{n}$ we get $(2) \triangleright u_{3},(3) \triangleright u_{4}, \ldots$, (1) $\triangleright u_{1},(l+1) \triangleright u_{2}, \ldots$, contrary to $G$ having finite global dimension.

Proposition $\mathrm{L}_{n}\left(\mathrm{~L}_{1}\right.$ is trivial) has a left and a right version. If $u=v q,|q| \geqslant 1$, factor $q$ by $\mathrm{U}_{n}$ into $q=a_{1} \cdots a_{l}$. Then segmentation assures us for suitable $s$ and $t$ 
that $v=\rightarrow s \rightarrow t=\rightarrow a_{1}$, whence by interpolation we get $v=\rightarrow s \rightarrow s t=u$. Likewise, if $u=q v$, we write $q=b_{1} \cdots b_{m}$, yielding by $\mathrm{S}_{n}$ and $\mathrm{I}_{n}$ the relation $b_{m}=\rightarrow s$ $\rightarrow s t=u \rightarrow t=\rightarrow v$. Clearly $u \rightarrow v$.

Finally, to check proposition $\mathrm{R}_{n}$, let $w \in W$ with $|w|=n$. Our proof of unique factorization actually applies to elements of $W$ as well as $M$ since we only use that subwords of $w$ lie in $M$. The proper conclusion is that $w \in W$ is either a "would-beatom" or has a unique factorization into genuine atoms.

The latter possibility leads to a contradiction when we write $w=a_{1} \cdots a_{m}$, with $a_{m}=\rightarrow a_{m-1}=\rightarrow \cdots=\rightarrow a_{1}$ in $A_{n-1}$. Since $m \geqslant 2$ is being assumed, this says (1) $\triangleleft a_{m}$. The relation $a_{1}=a_{m}$ would imply the impossibility $a_{1} \rightarrow a_{1}$, so $a_{m} \rightarrow a_{1}$ and chain extension forces (2) $\triangleleft a_{1}$. Letting $c a_{1}$ be an $l$-chain $(l \geqslant 2)$ whose tail has $a_{1}$ as a right factor, $c a_{1} a_{2} \cdots a_{m}$ is an $(l+1)$-prechain $(l+1 \geqslant 3)$ which implies (3) $\triangleright a_{i}$ and by $\mathrm{F}_{n}(3) \triangleleft a_{i}$ for some $a_{i}$. If $a_{i}=a_{1}$, repeat the argument to obtain (4) $\triangleleft a_{j}$ for some $a_{j}$, and so on. If instead $a_{i} \rightarrow a_{1}, \mathrm{C}_{n}$ yields (4) $a_{1}$. Since this construction could be iterated indefinitely, we have our desired contradiction.

THEOREM 6. Let $G$ be a connected graded monomial $\mathbf{k}$-algebra with global dimension $d<\infty$, such that $G$ contains no free subalgebra on two monomials. Then $G$ is finitely presented and has polynomial growth. Furthermore,

$$
H_{G}(z)=\prod_{i=1}^{d}\left(1-z^{e_{i}}\right)^{(-1)}
$$

for some positive integers $\left\{e_{i}\right\}$, so $H_{G}(z)$ is a rational function with a pole of order $d$ at $z=1$.

Proof. Suppose that the set $A$ of atoms for $G$ were infinite. The set $A$ is totally ordered, so choose any sequence $a_{1} \rightarrow a_{2} \rightarrow \cdots \rightarrow a_{d+1}$. We deduce (1) $\triangleright a_{2}$, (2) $\triangleright a_{3}, \ldots,(d) \triangleright a_{d+1}$, contradicting the requisite nonexistence of $d$-chains when $G$ has global dimension $d$.

The set of atoms for $G$ must be finite. In fact, if $D=\#(A)$, then $D \leqslant d$. The subset $S=A_{1} \subseteq A$ is also finite, so $G$ is finitely generated. By property $\mathrm{R}_{n}$ every $w \in W$ is the product of two atoms, so there can be only finitely many words in $W$. We have shown that $G$ is finitely presented, and by Theorem 3 it enjoys polynomial growth.

It turns out that there are precisely $d$ atoms. This is easiest to see by using the set of atoms as a set of generators. Call the new generating set $\left\{y_{1}, y_{2}, \ldots, y_{D}\right\}$, ordered so that $y_{i} \rightarrow y_{j}$ for $i>j$. By unique factorization and our knowledge about the relations in $W$ we know that a $\mathbf{k}$-basis for $G$ is the set

$$
\left\{y_{1}^{i_{1}} y_{2}^{i_{2}} \cdots y_{D}^{i_{D}}\right\}
$$

and consequently the "obstruction set" in the language of [2], relative to the generating set $\left\{y_{1}, y_{2}, \ldots, y_{D}\right\}$, is precisely $\left\{y_{i} y_{j} \mid i>j\right\}$. The longest chain constructible from these obstructions is clearly the $(D-1)$-chain $y_{D} y_{D-1} \cdots y_{1}$, so by [2, Theorem 1.4], $d=\operatorname{gl} . \operatorname{dim} .(G) \leqslant D$. Since $D \leqslant d$ also, we have $D=d$. 
Lastly, the $\mathbf{k}$-basis $\left\{y_{1}^{i_{1}} y_{2}^{i_{2}} \cdots y_{D}^{i_{D}}\right\}$ shows that $G$ 's Hilbert series is

$$
H_{G}(z)=\prod_{i=1}^{D}\left(1-z^{\left|y_{i}\right|}\right)^{(-1)},
$$

as claimed, where the absolute value symbol in (6) now refers to the original grading on $G$ if this differs from the grading by length.

EXAMPLE 3. It should be pointed out that monomial algebras with global dimension $d$ and polynomial growth do exist for every $d \geqslant 2$. While

$$
\mathbf{k}\left\langle x_{1}, \ldots, x_{d}\right\rangle /\left\langle\text { all } x_{i} x_{j} \mid i<j\right\rangle
$$

is an "obvious" example, we can achieve the same result with just two generators via

$$
\mathbf{k}\langle a, b\rangle /\left\langle a^{d-1} b, a^{i} b a^{j} b \mid 0 \leqslant j<i \leqslant d-2\right\rangle .
$$

The set of atoms for the latter example is $\left\{a, a^{i} b \mid 0 \leqslant i \leqslant d-2\right\}$. "More interesting" examples can be built by extending a set of generators to a suitable set $A$ of $d$ words to serve as the atoms, choosing a total ordering on $A$ which is consistent with the interpolation principle and with the membership of $A$, and taking as $W$ the set

$$
W=\{u v \mid u \in A, v \in A, u \rightarrow v, u v \notin A\} .
$$

For instance, in $\mathbf{k}\langle a, b\rangle$ the set $A=\left\{a, b, a b, a^{2} b, a b^{2}, a^{2} b^{2}\right\}$ works. The presence of $a b \in A$ indicates that we have taken $a \rightarrow b$. Note that interpolation forces all the other order relationships with the exception that either $a b \rightarrow a^{2} b^{2}$ or $a^{2} b^{2} \rightarrow a b$ is permissible.

REMARK. The Hilbert series (5) is identical with the Hilbert series of a commutative polynomial ring having generators in degrees $e_{1}, e_{2}, \ldots, e_{d}$. It also coincides with the Hilbert series of the enveloping algebra of a $d$-dimensional nilpotent graded Lie algebra $L$ for which $L=\operatorname{span}\left\{\alpha_{1}, \ldots, \alpha_{d}\right\}$ and $\left|\alpha_{i}\right|=e_{i}$. The polynomial ring and the enveloping algebra both have global dimension $d$. These observations make the following conjecture seem plausible.

CONJECTURE. Suppose $G$ is a connected graded $\mathbf{k}$-algebra with polynomial growth and with global dimension $d<\infty$. Then the Hilbert series of $G$ is given by (5) for some positive integers $\left\{e_{i}\right\}$.

The conjecture has now been verified when $G$ is commutative, when $G$ is an enveloping algebra, and when $G$ is a monomial ring. The referee has pointed out that an unpublished result of $\mathrm{M}$. Lorenz confirms the conjecture also in the case when $G$ is a Noetherian PI-ring.

4. One-two Hopf algebras with few relations. We will prove in this section that a connected graded Hopf algebra over an algebraically closed field, with $n$ generators in degree one and $d$ relations in degree two, contains a free subalgebra on two primitive elements as long as $d \leqslant \frac{1}{2}(3 n-5)$. As we noted in the Introduction, this is the case which holds the greatest relevance to local rings.

The proof of the existence of such a subalgebra is divided into five steps, each of which builds upon the previous one. Because of this, the motivation for some of the steps may seem obscure. It may help to read the proofs in reverse order, starting with Theorem 8 and working backwards to Lemma 2. 
As background, recall from [1, Lemma 1.1] that any finitely generated connected graded $\mathbf{k}$-algebra $G$ has a natural $\mathbf{k}$-basis consisting of monomials in the generators. Specifically, suppose $G$ is generated by $\left\{x_{1}, x_{2}, \ldots, x_{n}\right\}$, so that there exists a surjection of algebras $f: F=\mathbf{k}\left\langle x_{1}, \ldots, x_{n}\right\rangle \rightarrow G$. Let $T$ be the free noncommutative monoid on the graded set $S=\left\{x_{1}, \ldots, x_{n}\right\}$, and suppose that $S$ is totally ordered by a relation $>_{0}$. The degree and dictionary order on $T$ (induced by $>_{0}$ ) is defined by setting $u>v$ for $|u|>|v|$ and by resorting to the lexicographic order induced by $>_{0}$ when $|u|=|v|$. When $T$ has the degree and dictionary order, the set

$$
M_{G}=\{u \in T \mid f(u) \text { does not belong to } \operatorname{span}(f(v) \mid v<u)\}
$$

is a k-basis for $G$. Furthermore, $M_{G}$ is an order ideal of monomials, that is, any segment of any $u \in M_{G}$ also belongs to $M_{G}$. If $P$ is any further quotient of $G$, then $M_{P} \subseteq M_{G}$ (for details on any of this, see [1]).

Our goal, of course, is to establish the existence of suitable free subalgebras of a graded algebra. In a sense, the overall plan is to reduce this problem to a problem about monomial rings. Lemma 2 fits in with this by asserting that certain homomorphisms of graded monoids are well behaved.

Lemma 2. Let $T$ be the free monoid on the graded set $S=\left\{x_{1}, \ldots, x_{n}\right\}$. Let $S$ be totally ordered via $>_{0}$ and let $T$ inherit the degree and dictionary order, denoted $>$. Let $u$ and $v$ be any two words in $T$ and suppose that there exists no monomial $w$ of which both $u$ and $v$ are powers. Denote by $S^{\prime}$ the graded set $\{a, b\}$ having $|a|=|u|$ and $|b|=|v|$, and define $a>_{1} b$ if $u v>v u$ in $T, b>_{1} a$ if $v u>u v(u v=v u$ is ruled out by $\left[6, p\right.$. 237]). Let $T^{\prime}$ be the free monoid on $S^{\prime}$ and give $T^{\prime}$ the degree and dictionary order induced by $>_{1}$. Then the homomorphism of monoids $h: T^{\prime} \rightarrow T$ defined by $h(a)=u$ and $h(b)=v$ is order-preserving. That is, $h(p)>h(q)$ in $T$ if and only if $p>q$ in $T^{\prime}$. In particular, $h$ is one-to-one (this last claim coincides with Exercise 1 of $[6, p .238])$.

Proof. The nonexistence of $w$ having both $u$ and $v$ as powers assures us that $1 \neq u \neq v \neq 1$, so $|u|+|v| \geqslant 2$. Our proof proceeds by induction on $|u|+|v|$, so we suppose that $|u|+|v|=m \geqslant 2$ and that Lemma 2 is known to hold for all pairs of monomials $u^{\prime}, v^{\prime} \in T$ satisfying $\left|u^{\prime}\right|+\left|v^{\prime}\right| \leqslant m-1$.

The statement we wish to prove has the form that a certain relation holds for all monomials $p, q \in T^{\prime}$, and two cases are covered by an induction on $|p|$. They are

(I) the case where there is a subset $\{s, t\} \subseteq S$ for which $u=t s$ and $v=t$; and

(II) the case where neither $u$ nor $v$ is a left segment of the other.

Suppose the inequality $h\left(p^{\prime}\right)>h\left(q^{\prime}\right)$ is known to hold for $\left|p^{\prime}\right|<l$ and $q^{\prime}<p^{\prime}$, but $p$ and $q$ now satisfy $|p|=l$ and $q<p$. If $|q|<l$, then the "degree" part of the "degree and dictionary" guarantees that $h(q)<h(p)$, so suppose $|q|=l$. If $p$ and $q$ both share the left factor $a$, say $p=a p^{\prime}$ and $q=a q^{\prime}$, then $p^{\prime}>q^{\prime}$ so $h(p)=u h\left(p^{\prime}\right)$ $>u h\left(q^{\prime}\right)=h(q)$, and likewise if $p$ and $q$ share the left factor $b$. When $p=a p^{\prime}$ and $q=b q^{\prime}$, the relation $p>q$ in the lexicographic ordering indicates that $a>{ }_{1} b$, and we handle (I) and (II) separately. For (I), the nonexistence of $w$ having both $t s$ and $t$ as powers means that $s \neq t$, and $a>_{1} b$ leads to $t s t>t t s$, implying $s>_{0} t$. This fact 
combines with $|s|+\left|p^{\prime}\right|=l-|t|=\left|q^{\prime}\right|$ and $t$ being a left factor of $h\left(q^{\prime}\right)$ to yield that $s h\left(p^{\prime}\right)>h\left(q^{\prime}\right)$, whence $h(p)=t \operatorname{sh}\left(p^{\prime}\right)>t h\left(q^{\prime}\right)=h(q)$. For (II), $a>{ }_{1} b$ signifies $u v>v u$, which by lexicographic order means $u u^{\prime}>v v^{\prime}$ whenever $u^{\prime}, v^{\prime} \in T$ and $\left|u u^{\prime}\right|=\left|v v^{\prime}\right|$. In particular, $h(p)=u h\left(p^{\prime}\right)>v h\left(q^{\prime}\right)=h(q)$. A similar argument holds if $p=b p^{\prime}$ and $q=a q^{\prime}$.

Cases not covered above all have one of $u$ and $v$ occurring as a left segment of the other, without loss of generality say $v$ is a left segment of $u$. Writing $u=v v_{0}$, we have that $\left|v_{0}\right| \geqslant 1$ since otherwise $u=v$, and also that $v$ and $v_{0}$ cannot both be powers of the same $w \in T$. Let $T^{\prime \prime}$ be the free monoid on the graded set $\{s, t\}$, where $|s|=\left|v_{0}\right|$ and $|t|=|v|$. Define $h_{0}: T^{\prime \prime} \rightarrow T$ to be a homomorphism of monoids, with $h_{0}(s)=v_{0}$ and $h_{0}(t)=v$. Since $\left|v_{0}\right|+|v|<m=|u|+|v|, h_{0}$ is order-preserving by our inductive hypothesis as long as the ordering on $\{s, t\}$ satisfies $s>_{2} t \Leftrightarrow v_{0} v>v v_{0}$. This in turn is equivalent to

$$
v_{0} v>v v_{0} \Leftrightarrow v v_{0} v>v v v_{0} \Leftrightarrow u v>v u \Leftrightarrow a>_{1} b .
$$

Define the monoid homomorphism $h_{1}: T^{\prime} \rightarrow T^{\prime \prime}$ by $h_{1}(a)=t s$ and $h_{1}(b)=t$, which is order-preserving by case (I) above. Since $h=h_{0} \circ h_{1}, h$ also preserves order.

To apply this to situations involving linear combinations of monomials, we have the next two lemmas. For $F=\mathbf{k}\left\langle x_{1}, \ldots, x_{n}\right\rangle$ with $x_{1}>_{0} x_{2}>_{0} \cdots>_{0} x_{n}$, the high term $q$ of any $w \in F-(0)$ is found by expressing $w$ in terms of the monomial k-basis for $F$, say

$$
w=\sum_{v \in T} c_{v} v, \quad c_{v} \in \mathbf{k}, \text { almost all } c_{v}=0 .
$$

Then $q$ is the largest monomial, in the sense of the degree and dictionary order, for which $c_{q} \neq 0$. We continue from the previous section the notation $\langle u, v\rangle$ for the set of all segments of words in the submonoid of $T$ which $u$ and $v$ generate.

LEMMA 3. Let $f: F=\mathbf{k}\left\langle x_{1}, \ldots, x_{n}\right\rangle \rightarrow G$ be a surjection of graded algebras and let $T$ and $M_{G}$ be as defined above. Suppose that $u, v \in T$ are such that $\langle u, v\rangle \subseteq M_{G}$, and suppose that $\alpha$ and $\beta$ are any two nonzero homogeneous elements of $F$ whose high terms are, respectively, $u$ and $v$. Suppose further that $u$ and $v$ are not both powers of the same monomial. Then $f(\alpha)$ and $f(\beta)$ generate a free subalgebra of $G$.

Proof. Let $T^{\prime}$ be the free monoid on $\{a, b\}$, where $|a|=|u|$ and $|b|=|v|$, and let $h: T^{\prime} \rightarrow T$ be the homomorphism of monoids satisfying $h(a)=u$ and $h(b)=v$. When we order $\{a, b\}$ such that $a>{ }_{1} b \Leftrightarrow u v>v u$, Lemma 2 shows that $h$ is order-preserving. Furthermore, $\operatorname{im}(h) \subseteq\langle u, v\rangle \subseteq M_{G}$.

Let $g: \mathbf{k}\langle a, b\rangle \rightarrow F$ be the homomorphism of graded algebras defined by $g(a)=\alpha$ and $g(b)=\beta$. Let $p \in \operatorname{ker}(f \circ g)$ and if $p \neq 0$ write

$$
p=\sum_{r \in T^{\prime}} c_{r} r, \quad c_{r} \in \mathbf{k},
$$

almost all $c_{r}$ being zero. Let $q$ be the high term of $p$ and note that $h(r)$ is the high term of $g(r)$ for any $r \in T^{\prime}$. Consequently $h(q)$ is the high term of $g(p)$. Since

$$
0=f(g(p))=\sum_{r \in T^{\prime}} c_{r} f(g(r)) \text {, }
$$


we have the relation

$$
f(h(q)) \in \operatorname{span}\left\{f(w) \mid w<h^{i}: ;\right.
$$

in $G$. This contradicts the definition of $M_{G}$ since $h(q) \quad \because \quad \cdots$ $=0$, which is the desired conclusion.

LEMMA 4. Let $F=\mathbf{k}\left\langle x_{1}, \ldots, x_{n}\right\rangle$ be a free primitive!: each $\left|x_{i}\right|=1$. Let $\alpha_{1}, \ldots, \alpha_{d}$ be a set of primitive ele $G=F /\left\langle\alpha_{1}, \ldots, \alpha_{d}\right\rangle$. Write

$$
\alpha_{p}=\sum_{i=1}^{n} \sum_{j=1}^{n} c_{i j p} x_{i} x_{j}
$$

in $F, 1 \leqslant p \leqslant d, c_{i j p} \in \mathbf{k}$.

(a) If $c_{11 p}=0$ and $c_{12 p}=0$ for all $p$, then the subalgchrs ar. nerated by $x_{1}$ and $\left[x_{1}, x_{2}\right]$ is free on two generators.

(b) If $c_{12 p}=0$ and $c_{13 p}=0$ for all $p$, then the subalgebra of $G$ generated by $\left[x_{1}, x_{2}\right]$ and $\left[x_{1}, x_{3}\right]$ is free on two generators.

Note. The notation $[u, v]$ denotes $u v-(-1)^{|u| \cdot|v|} v u$, which is primitive whenever $u$ and $v$ are homogeneous primitives. In particular, $\left[x_{i}, x_{j}\right]=x_{i} x_{j}+x_{j} x_{i}$ is primitive, and any primitive element of degree two in $F$ is a linear combination of $\left\{\left[x_{i}, x_{j}\right]\right\}$ and $\left\{x_{i}^{2}\right\}$.

Proof. Because the relations are primitive, $c_{12 p}=0$ implics $c_{21 p}=0$ and likewise $c_{13 p}=0$ carries with it $c_{31 p}=0$. Considering $G$ as an associative graded algebra we form a further quotient $P$ of it. In case (a), let

$$
P=\mathbf{k}\left\langle x_{1}, \ldots, x_{n}\right\rangle /\left\langle x_{2}^{2}, x_{3}, \ldots, x_{n}\right\rangle \approx \mathbf{k}\left\langle x_{1}, x_{2}\right\rangle /\left\langle x_{2}^{2}\right\rangle .
$$

The k-basis for the monomial algebra $P$ consists of all $x_{1}$ sis on $x_{1}$ and $x_{2}$ which exclude the segment $x_{2}^{2}$, so $\left\langle x_{1}, x_{1} x_{2}\right\rangle=M_{P} \subseteq M_{G}$. Deduci by Lemma 3 that $\mathbf{k}\left\langle x_{1},\left[x_{1}, x_{2}\right]\right\rangle$ embeds freely in $G$.

In case (b), take $P$ to be

$$
\begin{aligned}
P & =\mathbf{k}\left\langle x_{1}, \ldots, x_{n}\right\rangle /\left\langle x_{1}^{2}, x_{2}^{2}, x_{2} x_{3}, x_{3} x_{2}, x_{3}^{2}, x_{1} \ldots, x_{n}\right\rangle \\
& \approx \mathbf{k}\left\langle x_{1}, x_{2}, x_{3}\right\rangle /\left\langle x_{1}^{2}, x_{2}^{2}, x_{2} x_{3}, x_{3} x_{2}, x_{3}^{2}\right\rangle .
\end{aligned}
$$

This time $\left\langle x_{1} x_{2}, x_{1} x_{3}\right\rangle=M_{P} \subseteq M_{G}$. So in $G$ the subalgetra generated by $\left[x_{1}, x_{2}\right]$ and $\left[x_{1}, x_{3}\right]$ is free.

We have emphasized that each quadratic relation $\alpha_{p}$, is given in terms of $n^{2}$ coefficients $\left(c_{i j p}\right)$, with $c_{i j p}=c_{j i p}$ in the case of primitive relations. Let $A_{p}$ denote the $n \times n$ matrix whose $(i, j)$-entry is $c_{i j p}$. If $\Lambda$ is any $n \times n$ nonsingular matrix with entries in $\mathbf{k}, \Lambda$ can be viewed as expressing a linear change of cordinates among the generators $\left\{x_{1}, \ldots, x_{n}\right\}$ of $F=\mathbf{k}\left\langle x_{1}, \ldots, x_{n}\right\rangle$. The effeci of wis basis change on the quadratic expression $\alpha_{p}$ is described by the congruence trionation, $A_{p}$ goes to $\Lambda^{t} A_{p} \Lambda$. Our approach is to select $\Lambda$ so that the quadratic forms described by ( $\Lambda^{t} A_{p} \Lambda$ ) will satisfy the hypotheses of Lemma 4. The rex rait gives a sufficient condition for the existence of such a matrix $\Lambda$. 
THEOREM 7. Fix $d \geqslant 1$ and for $1 \leqslant p \leqslant d$ let

$$
\alpha_{p}=\sum_{i=1}^{n} \sum_{j=1}^{n} c_{i j p} x_{i} x_{j}
$$

be primitive quadratic elements in the free Hopf algebra $F=\mathbf{k}\left\langle x_{1}, \ldots, x_{n}\right\rangle,\left|x_{i}\right|=1$. Let $A_{p}$ be the matrix wtih $\left(A_{p}\right)_{(i, j)}=c_{i j p}$ and for any column vector $y=\left(y_{1}, \ldots, y_{n}\right)^{t}$ $\in \mathbf{k}^{n}$ let $C(y)$ denote the $d \times n$ matrix whose pth row is $y^{t} A_{p}$. Suppose that $y \in \mathbf{k}^{n}-(0)$ exists such that $\operatorname{rank}(C(y)) \leqslant n-2$. Then $G=F /\left\langle\alpha_{1}, \ldots, \alpha_{d}\right\rangle$ contains a free primitively generated subalgebra on two generators of degree one or two. In particular, this is true over any $\mathbf{k}$ if $d \leqslant n-2$.

Proof. Suppose $y$ is such that $y \neq 0$ and $\operatorname{rank}(C(y)) \leqslant n-2$. Let $z_{1}$ and $z_{2}$ be linearly independent vectors in $\mathbf{k}^{n}$ belonging to the null space of $C(y)$. Let $U$ denote the subspace of $\mathbf{k}^{n}$ spanned by $z_{1}$ and $z_{2}$.

If the vector $y$ does not belong to $U$, let $\Lambda$ be any nonsingular matrix whose first three columns are, respectively, $y, z_{1}$, and $z_{2}$. The $(1,2)$-entry of $\Lambda^{t} A_{p} \Lambda$ equals $y^{t} A_{p} z_{1}=0$ and likewise the (1,3)-entry of $\Lambda^{t} A_{p} \Lambda$ equals $y^{t} A_{p} z_{2}=0$. With respect to the new basis described by the columns of $\Lambda$, the quadratic relations defining $G$ all satisfy condition (b) of Lemma 4.

If instead $y \in U$, then $C(y) y=0$ and at least one of $z_{1}$ and $z_{2}$, say $z_{1}$, is not parallel to $y$. Let $\Lambda$ be any nonsingular matrix whose first two columns are, respectively, $y$ and $z_{1}$. The $(1,1)$-entry of $\Lambda^{t} A_{p} \Lambda$ equals $y^{t} A_{p} y=0$ and the $(1,2)$-entry is $y^{t} A_{p} z_{1}=0$. With respect to the new basis, the defining relations for $G$ satisfy condition (a) of Lemma 4.

THEOREM 8. Suppose $\mathbf{k}$ is an algebraically closed field. Let

$$
\alpha_{p}=\sum_{i=1}^{n} \sum_{j=1}^{n} c_{i j p} x_{i} x_{j}
$$

be primitive quadratic elements in $F=\mathbf{k}\left\langle x_{1}, \ldots, x_{n}\right\rangle$ for $1 \leqslant p \leqslant d$, where each $\left|x_{i}\right|=1$ and $d \leqslant \frac{1}{2}(3 n-5)$. Then $G=F /\left\langle\alpha_{1}, \ldots, \alpha_{d}\right\rangle$ contains a free subalgebra generated by two primitives of degree one or two.

Proof. Let $C(y)$ be as in Theorem 7, but think of the coordinates $y_{1}, \ldots, y_{n}$ of $y$ as indeterminates allowed to range over $\mathbf{k}$. The entries of $C(y)$ are now homogeneous linear forms in $\mathbf{k}\left[y_{1}, \ldots, y_{n}\right]$. If $d \leqslant n-2$ there is nothing to prove by Theorem 7 , so suppose $d \geqslant n-1$.

Let $I$ be the ideal in the polynomial ring $\mathbf{k}\left[y_{1}, \ldots, y_{n}\right]$ generated by the determinants of all $(n-1) \times(n-1)$ minors of $C(y)$. By [7 or 8, Corollary 4], the grade of $I$, which is the length of the longest regular sequence lying in $I$, is bounded by

$$
\operatorname{grade}(I) \leqslant(n-(n-1)+1)(d-(n-1)+1)=2 d-2 n+4 \leqslant n-1 .
$$

Let $V$ be the algebraic variety defined by $I$. If $V=\{0\}$, then the Nullstellensatz [3] would guarantee that $\operatorname{Rad}(I)=\left(x_{1}, \ldots, x_{n}\right)$, and sufficiently high powers of $x_{1}, \ldots, x_{n}$ would form a regular sequence lying in $I$. This contradiction shows that $V \neq\{0\}$, i.e., there is some $y^{\prime} \in \mathbf{k}^{n}-(0)$ for which every $(n-1) \times(n-1)$ minor of $C\left(y^{\prime}\right)$ 
has vanishing determinant. It follows that $\operatorname{rank}\left(C\left(y^{\prime}\right)\right) \leqslant n-2$, as needed for Theorem 7.

Applying [13 or 15], we have at once

THEOREM 9. Let $(R, \mathbf{m}, \mathbf{k})$ be a local ring with $\mathbf{m}^{3}=0, \operatorname{dim}\left(\mathbf{m} / \mathbf{m}^{2}\right)=n$, and $\operatorname{dim}\left(\mathbf{m}^{2}\right)=d$. If (a) $\mathbf{k}$ is arbitrary and $d \leqslant n-2$ or (b) $\mathbf{k}$ is algebraically closed and $d \leqslant \frac{1}{2}(3 n-5)$, then the subalgebra $E$ of $\operatorname{Ext}_{R}^{*}(\mathbf{k}, \mathbf{k})$ which is generated by $\operatorname{Ext}_{R}^{1}(\mathbf{k}, \mathbf{k})$ contains a free subalgebra on two primitive elements.

Appendix: Proof of interpolation. In this Appendix we present the proof of proposition $\mathrm{I}_{n}$ ( $\mathrm{I}_{1}$ is vacuous), taking as hypotheses that all ten propositions of Theorem 5 hold for $n-1$, that propositions $\mathrm{U}_{n}, \mathrm{~S}_{n}$, and $\mathrm{P}_{n}$ are true, and that the hypotheses of Theorem 5 are met by $W \subseteq T=T_{S}$.

Recall that, for monomials $s$ and $t, F_{s, t}$ denotes the submonoid of $T$ generated by $s$ and $t$ and $\langle s, t\rangle$ denotes the set of all segments of words in $F_{s, t}$. If $s, t \in M$, then any $w \in\langle s, t\rangle \cap W$ must have the form $w=w_{1} w_{2} w_{3}$, where $w_{2} \in F_{s, t}, w_{1}$ is a final segment of $s$ or of $t$, and $w_{3}$ is an initial segment of $s$ or of $t$.

ProOF OF INTERPOLATION. Interpolation provides a specific $\rightarrow$-connection between an atom of length $n$ and a shorter atom. The author initially attempted several proofs of Theorem 5 which avoided or glossed over this step, and all of them ran into trouble later with the chain extension property or with exclusivity. Half of the claim $\mathrm{I}_{n}$ is simple. If $v \rightarrow u v$, there would exist a word $w \in\langle u v, v\rangle \cap W$ having the form $w=s_{1} w^{\prime} s_{2}$, where $s_{1}$ is a nontrivial right factor of $v, w^{\prime} \in F_{u v, v}$, and $s_{2}$ is a left factor of $u v$ or of $v$. This word $w$ also shows that $v \rightarrow u$, contradicting exclusivity and the assumption $u \rightarrow v$. The relation $u v \rightarrow v$ follows, since the condition of no free subalgebras guarantees that one or the other must hold.

The proof that $u \rightarrow u v$ or that $u \rightarrow u^{\prime} \rightarrow u v$ is surprisingly more difficult. The difficulty reflects a basic asymmetry in our definition of the relation $\rightarrow$. Among all pairs $(s, t)$ of atoms in $A_{n-1}$, let $\left(u^{\prime}, u^{\prime \prime}\right)$ be the pair for which $\left|u^{\prime}\right|$ is largest. By $\mathrm{L}_{n-1}$ we have $u \rightarrow u^{\prime}$, so it suffices to prove that $u^{\prime} \rightarrow u^{\prime} u^{\prime \prime}=u v$. To simplify notation we henceforth assume that $u=u^{\prime}$ and proceed to prove that $u \rightarrow u v$.

Let

$$
\begin{array}{r}
B=\left\{a_{m} \mid w \text { is a proper nontrivial left factor of } u v \text { and } w=a_{1} \cdots a_{m}\right. \\
\text { is its unique monotonic factorization into atoms }\} .
\end{array}
$$

In words, $B$ consists of all final atoms of all proper initial segments of $u v$. Clearly $u \in B \subseteq A_{n-1}$.

Assertion 1. The atom $u\left(=u^{\prime}\right)$ is the latest element of $B$. (When $a_{2} \rightarrow a_{1}$ we say that $a_{1}$ is "later" than $a_{2}$.)

To prove this assertion, let $a_{1} \cdots a_{m}$ be the atomic factorization of any proper left segment of $u v$. If $|u| \geqslant\left|a_{1}\right|$ we have $a_{m}=\rightarrow a_{1}=\rightarrow u$ by $\mathrm{L}_{n-1}$. If $|u|<\left|a_{1}\right|$, we reach a contradiction as follows. Use $\mathrm{L}_{n-1}$ to deduce $u \rightarrow a_{1}$ and use $\mathrm{S}_{n}$ to obtain $a_{1}=\rightarrow s \rightarrow t$ with $s t=u v$. Then $u \rightarrow s$ so $\mathrm{L}_{n-1}$ shows $|u|<|s|$, making $(s, t)$ an atomic pair having $s t=u v$ but $|s|>|u|=\left|u^{\prime}\right|$. 
We shall also make use of the following three facts.

Assertion 2. If $r$ is a left segment of any $w \in F_{u, u v}$, then either $r \in F_{u, u v}$ or $r=r_{1} r_{2} r_{3}$, where $r_{1} \in F_{u, u v}$ and $r_{3} \in B$ satisfies $r_{3} \rightarrow u$.

Assertion 3. Let $b \in B-\{u\}$. If $r$ is a nontrivial left segment of any $w \in F_{b, u}$, then either $r=r_{1} u$ for some $r_{1} \in F_{b, u}$ or $r=r_{1} r_{2} r_{3}$, where $r_{1} \in F_{b, u}$ and $r_{3} \in B-$ $\{u\}$.

Assertion 4. Suppose $u v=u^{h} v^{\prime}$ for some $h \geqslant 0$. Then any proper left segment $v^{\prime \prime}$ of $v^{\prime}$ either has the form $v^{\prime \prime}=u^{m}$, where $u^{m}$ is also a left factor of $u v$, or has the form $v^{\prime \prime}=r_{1} r_{2}$ with $r_{2} \in B-\{u\}$.

To prove Assertion 2, note that $r=r_{1} r^{\prime}$, where $r_{1} \in F_{u, u v}$ and $r^{\prime}$ is a proper left factor of $u v$. If $r^{\prime}=1$ we are done, but when $r^{\prime} \neq 1$ factoring $r^{\prime}=a_{1} \cdots a_{m}$ by $\mathrm{U}_{n-1}$ yields $a_{m} \in B$ and, by Assertion $1, a_{m}=\rightarrow u$. Either $a_{m} \rightarrow u$ and we are done (take $r_{3}=a_{m}$ ), or $a_{m}=u$. If $a_{m}=u$, then $u=a_{m}=\rightarrow \cdots=\rightarrow a_{1}=\rightarrow u$ by Assertion 1, so $u=a_{m}=\cdots=a_{1}$ and $r^{\prime}=u^{m}$. But then $r=r_{1} r^{\prime} \in F_{u, u v}$.

The proof of Assertion 3 is very similar, but it relies in addition upon the fact that the final atom of an initial segment of any $b \in B$ also lies in $B$. This fact is a consequence of the "observation" made while proving proposition $\mathrm{U}_{n}$.

To prove Assertion 4, factor $v^{\prime \prime}$ by $\mathrm{U}_{n-1}$ into $v^{\prime \prime}=a_{1} \cdots a_{m}$. If $a_{1}=\rightarrow u$, then $u^{h} a_{1} \cdots a_{m}$ is the unique factorization of $u^{h} v^{\prime \prime}$, so $a_{m} \in B$. When $a_{m}=u$, we get $v^{\prime \prime}=u^{m}$ and since $u^{m+h}$ is then a left factor of $u^{h} v^{\prime}=u v, u^{m}$ is also. If instead $u \rightarrow a_{1}$, then $h \geqslant 1$ by Assertion 1. Proposition $\mathrm{I}_{n-1}$ makes $u^{h} a_{1}$ an atom and by $\mathrm{S}_{n}$ and $\mathrm{L}_{n-1}$ we have $u^{h} a_{1} \rightarrow s \rightarrow t$ with $|s|>|u|$, contradicting the maximality of $|u|=\left|u^{\prime}\right|$.

With these four assertions behind us, we now prove that $u \rightarrow u v$ by supposing the opposite and reaching a contradiction. Using this supposition and the fact that " $v \rightarrow u$ " is false by $\mathrm{E}_{n-1}$, we may conclude

Assertion 5. Every word $w$ in $W \cap\langle u, u v\rangle$ must have the form $w=s_{1} v w^{\prime} s_{2}$, where $s_{1} \neq 1$ is a right factor of $u, w^{\prime} \in F_{u, u v}$, and $s_{2}$ is a left factor of $u$ or of $u v$.

Using Assertion 5, we obtain the contradiction we seek by proving Assertion 6. There is a set $Q \subseteq M$ with the following two properties:

(A) For some $m \geqslant 0$, there is an $m$-chain whose tail belongs to $Q$.

(B) For all $m \geqslant 0$, if there exists an $m$-chain whose tail $q$ belongs to $Q$, then for some $l>m$ there is an $l$-chain whose tail $q^{\prime}$ also belongs to $Q$.

Obviously, if Assertion 6 were true, then there would exist arbitrarily long chains and by Theorem 4 gl.dim. $(\mathbf{k}\langle S\rangle /\langle W\rangle)=\infty$. This would contradict the hypotheses of Theorem 5. After giving names to four special words in $W$, we shall define a set $Q$ for which we will be able to prove (A) and (B).

Let $w_{0}$ be a word of minimal length in the set $W \cap\langle u, u v\rangle$. If $B$ consists of $\{u\}$ only, we can proceed immediately to define $Q$. If instead $B$ contains two or more elements, let $b$ denote the latest element of $B-\{u\}$. There may or may not exist a word in $W$ of the form $s w^{\prime} u v$, where $s$ is a nontrivial right factor of $b$ and $w^{\prime} \in F_{b, u}$. If so, let $w_{1}$ have mimimal length among words of this form. Let $w_{2}$ be a word of minimal length, if any exist, such that $w_{2}=s w^{\prime} u v$, where as before $w^{\prime} \in F_{b, u}$ but $s$ is now a nontrivial right factor of $u$. Lastly, let $w_{3}$ be of minimal length in $W \cap\langle b, u\rangle$. 
Let $Q=Q_{1} \cup Q_{2} \cup Q_{3} \cup Q_{4}$ if $B \neq\{u\}$ and set $Q=Q_{4}$ if $B=\{u\}$, where $Q_{j}$ denotes the set of words $q \in M-\{1\}$ which satisfy condition $(j)$ below ( $\left|w_{i}\right|$ is taken to be $\infty$ if $w_{i}$ does not exist):

(1) Some $a \in B-\{u\}$ is a right factor of $q$.

(2) $q=t u^{h}$, where $t \in F_{b, u} ; u^{h}$ is a nontrivial left factor of $u v$; and

$$
|t|<\min \left(\left|w_{1}\right|-|u v|, \max \left(\left|w_{3}\right|-|u|,\left|w_{2}\right|-|u v|\right)\right) .
$$

(3) $q=t u v$, where $t \in F_{b, u}$.

(4) $q=v^{\prime} t$, where $v^{\prime}$ is any right factor of $v$ and $t \in F_{u, u v}-\{1\}$ and $|t|<\left|w_{0}\right|-|v|$.

To prove that Assertion 6(A) holds for this $Q$, consider $|u|=1$ and $|u|>1$ separately. If $|u|=1$, then $u$ is itself a 0 -chain with tail $u$, so $u \in Q_{4}$. If $|u|>1$, let $a \in S$ be the first letter of the word $u$. Then $a$ is a 0 -chain whose tail is $a \in Q_{1}$.

To verify the inductive step of Assertion 6, some additional notation is helpful. Let $c$ be an $m$-chain with tail $q$, and suppose that $w \in W$ is any word such that a nontrivial initial factor $r$ of $w$ is also a final factor of $q$. Write $w=r w^{\prime}$ and note that $c w^{\prime}$ is an $(m+1)$-prechain. This prechain has as a left factor an $(m+1)$-chain $c^{\prime}$ with tail $q^{\prime}$. The new tail $q^{\prime}$ is defined to be the unique initial segment of $w^{\prime}$ such that some right factor of $q q^{\prime}$ belongs to $W$ but no other segment of $q q^{\prime}$ lies in $W$. Under these conditions we write $q^{\prime}=\mathrm{NT}\left(q, w^{\prime}\right)$, "NT" standing for "new tail". As we have already noted, $\mathrm{NT}\left(q, w^{\prime}\right)$ is always a nontrivial left factor of $w^{\prime}$.

We are finally ready to verify Assertion 6(B), which is the heart of the proof of interpolation. Suppose $c$ is an $m$-chain with tail $q$ satisfying (1). By $\mathrm{C}_{n-1}$ and $\mathrm{F}_{n-1}$ we can immediately reduce to the case $a=b$. Consider two subcases. If $\left|w_{3}\right|<\left|w_{1}\right|-|v|$, write $w_{3}=s_{1} s_{2} s_{3}$, where $s_{2} \in F_{b, u}$ and $s_{1} \neq 1$ is a final factor of $b$. Put $q^{\prime}=$ $\operatorname{NT}\left(q, s_{2} s_{3}\right)$. By Assertion 3, either $q^{\prime} \in Q_{1}$ or $q^{\prime}=t u$ for some $t \in F_{b, u}$. In the latter event,

$$
|t|+|u|=\left|q^{\prime}\right| \leqslant\left|s_{2} s_{3}\right|<\left|w_{3}\right|<\left|w_{1}\right|-|v|,
$$

so $|t|<\left|w_{3}\right|-|u|<\left|w_{1}\right|-|u v|$ and consequently $q^{\prime} \in Q_{2}$.

If instead $\left|w_{3}\right| \geqslant\left|w_{1}\right|-|v|$, let $w_{1}=s_{1} s_{2} u v$, where again $s_{1} \neq 1$ is a final factor of $b$ and $s_{2} \in F_{b, u}$. Put $q^{\prime}=\mathrm{NT}\left(q, s_{2} u v\right)$. Since $q^{\prime}$ is an initial segment of $s_{2} u v$, we must have $q^{\prime}=s_{2} u v$ or $\left|q^{\prime}\right| \leqslant\left|s_{2}\right|$ or $\left|s_{2} u v\right|>\left|q^{\prime}\right|>\left|s_{2}\right|$. If $q^{\prime}=s_{2} u v$, then $q^{\prime} \in Q_{3}$, while $\left|q^{\prime}\right| \leqslant\left|s_{2}\right|$ means by Assertion 3 that either $q^{\prime} \in Q_{1}$ or $q^{\prime}=t u$ with $t \in F_{b, u}$. Lastly, if $\left|s_{2} u v\right|>\left|q^{\prime}\right|>\left|s_{2}\right|$, then Assertion 4 shows that either $q^{\prime} \in Q_{1}$ or $q^{\prime}=s_{2} u^{h}$ for $u^{h}$ a left segment of $u v$. When $q^{\prime} \notin Q_{1} \cup Q_{3}$ we have $q^{\prime}=t u^{h}$ with

$$
|t| \leqslant\left|s_{2}\right|<\left|w_{1}\right|-|u v| \leqslant\left|w_{3}\right|-|u|
$$

so $q^{\prime} \in Q_{2}$.

If we start with $q \in Q_{2}$, write $q=t u^{h}$ and again consider two subcases. If $|t| \geqslant\left|w_{2}\right|-|u v|$, factor $w_{2}$ as $w_{2}=s_{1} s_{2} u v$, where $s_{2} \in F_{b, u}$ and $s_{1} \neq 1$ is a right factor of $u$. Put $q^{\prime}=\mathrm{NT}\left(q, s_{2} u v\right)$. By reasoning similar to that of the previous paragraph we have either $q^{\prime} \in Q_{1} \cup Q_{3}$ or $q^{\prime}=t^{\prime} u^{m}$ with $\left|t^{\prime}\right| \leqslant\left|s_{2}\right|$. Then

$$
\left|t^{\prime}\right| \leqslant\left|s_{2}\right|<\left|w_{2}\right|-|u v| \leqslant|t|<\left|w_{1}\right|-|u v| \text {, }
$$

so $q^{\prime} \in Q_{2}$. 
If instead $|t|<\left|w_{2}\right|-|u v|$, use Assertion 5 to factor $w_{0}$ as $w_{0}=s_{1} v s_{2} s_{3}$, where $s_{1}$ is a right factor of $u, s_{2} \in F_{b, u}$, and $\left|s_{3}\right|<|u v|$. Write $u v=u^{h} v^{\prime}$ (where $h$ is still such that $\left.q=t u^{h}\right)$ and put $\because^{\prime}=\mathrm{NT}\left(q, v^{\prime} s_{2} s_{3}\right)$. Consider the possibilities of $\left|q^{\prime}\right|<\left|v^{\prime}\right|$ and $\left|q^{\prime}\right| \geqslant\left|v^{\prime}\right|$ separately. When $\left|q^{\prime}\right|<\left|v^{\prime}\right|$, Assertion 4 shows $q^{\prime} \in Q_{1} \cup Q_{2}$. When $\left|q^{\prime}\right| \geqslant\left|v^{\prime}\right|$, Assertion 2 assures us that $q^{\prime} \in Q_{1}$ or $q^{\prime}=v^{\prime} t^{\prime}$ with $t^{\prime} \in F_{u, u v}$. In the latter event we have $\left|t^{\prime}\right| \leqslant\left|s_{2} s_{3}\right|<\left|w_{0}\right|-|v|$, so $q^{\prime} \in Q_{4}$ as long as we can show that $t^{\prime} \neq 1$. If $t^{\prime}=1$ there would exist a word $w \in W$ which was a final segment of $q q^{\prime}=t u^{h} v^{\prime}=t u v$. Because of the special form of $w$ and the minimality of $w_{1}$ and of $w_{2}$, we would deduce that either $|w| \geqslant\left|w_{1}\right|$ or $|w| \geqslant\left|w_{2}\right|$. If $|w| \geqslant\left|w_{1}\right|$ we obtain the contradiction

$$
\left|w_{i}\right| \leqslant|w| \leqslant\left|q q^{\prime}\right|=|t u v|=|t|+|u v|<\left|w_{1}\right|
$$

while if $|w| \geqslant\left|w_{2}\right|$ we deduce the absurdity

$$
\left|w_{2}\right| \leqslant|w| \leqslant\left|q q^{\prime}\right|=|t u v|<\left|w_{2}\right| \text {. }
$$

Next suppose th: $\quad l u v \in Q_{3}$. Again write $w_{0}=s_{1} v s_{2} s_{3}$, where $s_{1} \neq 1$ is a right segment of $u$ and $\quad \operatorname{NT}\left(q, s_{2} s_{3}\right)$. Because $\left|q^{\prime}\right| \leqslant\left|s_{2} s_{3}\right|<\left|w_{0}\right|-|v|$, Assertion 2 guarantees that $q^{\prime} \therefore \quad:$

Lastly, if $q \in \ldots$. We can also factor $t$ as $t=t_{1} t_{2}$, where $\quad \cdots \quad$ or $t_{2}=u$ or $t_{2}=u v$. If $t_{2}=u v$, write $w_{0}=s_{1} v s_{2} s_{3}$ and put $q^{\prime}=\mathrm{NT}\left(q_{i} \ldots Q_{4}\right.$ as in the previous paragraph. If $t_{2}=u$, let $q^{\prime}=$ $\mathrm{NT}\left(q, v s_{2} s_{3}\right)$. A ad 4 show that $q^{\prime} \in Q_{1} \cup Q_{4} \cup\{v\}$. But $q^{\prime}=v$ leads to a contradict. $\quad \cdots$ s. If $q^{\prime}=v$, then $q q^{\prime}=v^{\prime} t_{1} u v$ has a final segment $w \in W$. Becaus $\quad\rangle$ we have $w \in W \cap\langle u, u v\rangle$ and therefore $|w| \geqslant\left|w_{0}\right|$. Since $|t|<\left|w_{0}\right| \cdots|t v|<\left|w_{0}\right| \leqslant|w|$, so $w=v^{\prime \prime} t v$ for some nontrivial right factor $v^{\prime \prime}$ of $v$. Bill ins, ,..: u (cf. Assertion 5), which is impossible.

\section{REFERENCES}

1. D. J. Anick, Yine graded algebras and their Hilbert series, J. Algebra 78 (1982), $120-140$.

2. On the On ociative algebras, Trans. Amer. Math. Soc. (to appear).

3. M. F. Atiyah an! । i sonald, Introduction to commutative algebra, Addison-Wesley, Reading, Mass., 1969.

4. L. Avramov, fice ta the cohomology of local rings, Trans. Amer. Math. Soc. 270 (1982), 589-608.

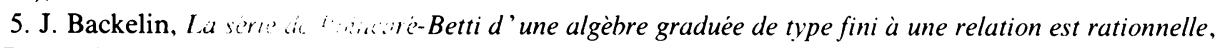
C. R. Acad. Sci. Paris $4 . .$.

6. P. M. Cohn, Free H? 'me' heir relations, London Math. Soc. Monographs No. 2, Academic Press, London and New York $1, ; i$

7. J. A. Eagon, Ideals gericrated hy the subdeterminants of a matrix, Thesis, Univ. of Chicago, 1961.

8. J. A. Eagon and M. Hochster. Cohen-Macaulay rings, invariant theory, and the generic perfection of determinental loci, Amer. J. Math. 93 (1971), 1020-1058.

9. Y. Felix and S. Halperin, Rational L.-S. category and its applications, Trans. Amer. Math. Soc. 270 (1982), 577-588.

10. Y. Felix and J. C. Thomas, Characterization of spaces whose rational category is two, preprint. 
11. E. S. Golod and I. R. Shafarevich, On nil algebras and finitely approximable groups, Izv. Akad. Nauk SSSR Ser. Mat. 28 (1964), 273-276.

12. J.-M. Lemaire, Algèbres connexes et homologie des espaces de lacets, Lecture Notes in Math., vol. 422, Springer-Verlag, Berlin and New York, 1974.

13. C. Löfwall, On the subalgebra generated by the one-dimensional elements in the Yoneda Ext-algebra, Reports No. 5, Matematiska Inst., Stockholms Universitet, Sweden, 1976.

14. J. Milnor, A note on curvature and fundamental group, J. Differential Geom. 2 (1968), 1-7.

15. J.-E. Roos, Relations hetween Poincaré-Betti series of loop spaces and of local rings, Lecture Notes in Math., vol. 740, Springer-Verlag, Berlin and New York, 1979, pp. 285-322.

Department of Mathematics, Massachusetts institute of Technology, Cambridge, MasSACHUSETTS 02139 